\title{
Líderes que quieren marcar agenda. Nuevas derivaciones de la Agenda Setting
}

\author{
Daniel Cabrera ${ }^{1}$
}

Fecha de recepción: 2 de octubre de 2016

Fecha de aprobación: 30 de noviembre de 2016

\begin{abstract}
Resumen
El traslado de la relevancia temática de medios a público ha sido el eje principal del análisis propuesto por las investigaciones en agenda setting. Basado en una investigación empírica, sostenida en una encuesta que abarcó 643 casos representativos de los ciudadanos residentes en la Ciudad de Buenos Aires, y en el relevamiento de 20.000 noticias periodísticas, este artículo presenta datos que no alcanzan para sostener la hipótesis, pero tampoco para rechazarla. Por otra parte, se describe la composición de un público social, económica y políticamente compacto y muy proclive a aceptar el mensaje mediático. A la vez, abre la puerta para la introducción de nuevos factores contingentes en el estudio del efecto agenda y retoma la hipótesis de la comunicación en dos pasos, en la que líderes políticos juegan un papel relevante.
\end{abstract}

Palabras clave: agenda setting, medios de comunicación, opinión pública, líderes

\section{Leaders who want to set agenda. Our referrals of the Setting Agenda}

\begin{abstract}
The transfer of the thematic relevance of media to public has been the main axis of the analysis proposed by the researches in setting agenda. Based on an empirical investigation, supported by a survey that included 643 representative cases of the citizens residing in the city of Buenos Aires, and in the study of 20.000 journalistic news, this article presents data that aren't enough to sustain the hypothesis, but neither to reject it. On the other hand, it describes the composition of a social public, economically and politically small, and very prone to accept the media message. At the same time, it opens the door to the introduction of new contingent factor son the study of the agenda ef-
\end{abstract}

Licenciado en Ciencia Política (UBA), Magíster en Análisis de la Opinión Pública (UNSAM), Docente e investigador de la Facultad de Ciencias Sociales, Universidad de Buenos Aires, Argentina. Contacto: dcabrera@fibertel.com.ar. 
fect, and takes up the hypothesis of communications in two steps, in which the political leaders play a relevant role.

Keywords: setting agenda, mass media, public opinion, leaders

\title{
Líderes que querem marcar agenda. Novas derivações da Agenda Setting
}

\begin{abstract}
Resumo
O traslado da relevância temática dos médios a públicos tem sido o foco principal da análise proposta pelas pesquisas em agenda setting. Baseado numa pesquisa empírica, sustentada numa indagação abrangendo 643 casos representativos dos cidadãos que vivem na cidade de Buenos Aires, e o relevo de 20.000 notícias jornalísticas, este artigo apresenta os dados que não alcançam o sustento para apoiar a hipótese, mas também não para rejeitála. Além disso, descreve-se a composição de um público social, econômico e politicamente compacto e muito propenso a aceitar a mensagem da mídia. Por sua vez, abre a porta para a introdução de novos fatores contingentes no estudo do efeito agenda e retoma a hipótese da comunicação em duas etapas, onde líderes políticos desempenham um papel importante.
\end{abstract}

Palavras-chave: agenda setting, médios de comunicação, opinião pública, líderes.

\section{Introducción}

En los últimos años prolifera en Argentina una discusión acerca del rol social y político de los medios de comunicación masivos. La polémica abarca grandes segmentos sociales, aunque cobró fuerza en ámbitos políticos y académicos, especialmente a partir de 2008, cuando el conflicto entre el gobierno nacional de entonces y sectores rurales tuvo una derivación algo inesperada, iniciando una fuerte controversia que se extiende hasta la actualidad entre medios denominados tradicionales o hegemónicos ${ }^{2}$ -principalmente el grupo Clarín y el diario La Nación- y el gobierno presidido por Cristina Fernández de Kirchner (Kitzberger, 2012; Zunino, 2014).

El contrapunto tuvo a su vez una arista legislativa y judicial, al sancionarse en 2009 la denominada Ley de Servicios de Comu-

2 Las denominaciones corren por cuenta de los contendientes. 
nicación Audiovisual N ${ }^{\circ}$ 26.522, objetada por el grupo Clarín y dirimida en la instancia jurídica más elevada.

La pugna puede leerse como una simple continuidad de las críticas mediáticas hacia los gobernantes, aunque habría que reclamar para el episodio un lugar preponderante, análogo por su intensidad, aunque no por sus derivaciones, a los conflictos que tuvieron lugar durante el gobierno del presidente Menem con el diario Página 12; durante la administración Alfonsín también con el grupo Clarín; en los últimos años de la dictadura militar con las publicaciones de editorial La Urraca, sobre todo con la revista Humor, y, para no retroceder demasiado en la historia, al finalizar el gobierno del derrocado presidente Illia con las revistas Primera Plana, Todo y Confirmado.

No obstante, el conflicto actual ocupa un lapso inédito e imprevisto, aunque el cambio de gestión gubernamental en 2015 aplacó la fuerza de uno de los antagonistas al ser derrotado el kirchnerismo en las elecciones presidenciales. Por otra parte, si bien la reyerta se explica en algunos círculos a partir de ciertos desacuerdos económicos entre las partes ${ }^{3}$, en la superficie la discrepancia expone un cuestionamiento que gira alrededor de la posible influencia de los medios sobre el público, que los adversarios exacerban, por un lado, otorgando a los medios un poder casi ilimitado para manipular opiniones y comportamientos (Cabrera, 2011) y, por otro, exhibiéndose como meros y objetivos reflejos de los intereses de las audiencias, a las que solo les importa la verdad.

Sin embargo, la relevancia del tema es relativa, ya que pareciera ser que el cuestionamiento a los medios -especialmente durante las campañas electorales- proviene fundamentalmente de un público politizado, lo que se apoya en la idea de que "el

Pueden tomarse como un indicio al respecto las palabras de Julio Blanck en la entrevista que le realizó Fernando Rosso para La izquierda diario: Recurso electrónico disponible en: http://www.laizquierdadiario.com/Julio-Blanck-EnClarin-hicimos-un-periodismo-de-guerra. 
poder de los medios reposa no tanto en su influencia (...) sobre el público sino en la percepción que los expertos y los decisores de políticas tienen sobre tal influencia..." (Walgrave y Van Aeslst, en Aruguete, 2015, p. 116) debido a que, en palabras de otros autores, "los medios parecen hablarse a sí mismos y a las elites políticas y económicas y no a su amplia base de consumidores" (Boczkowski y Mitchelstein, 2015, p. 50).

\section{Estableciendo la agenda}

En este marco, la investigación sobre la que se basa este artículo abordó una de las hipótesis más firmes relacionadas con la influencia de los medios ${ }^{4}$.

La llamada "teoría de la agenda setting" (McCombs y Shaw, 1972; Casermeiro, 2003; McCombs, 2006 y 2010; Aruguete, 2015) -un hito en la historia de la investigación acerca de la relación entre medios y audiencias- surge luego de un primer ciclo en el estudio de los medios, en el que se postulaba una muy poderosa, directa e indiscriminada influencia de estos sobre los comportamientos sociales mediante "irresistibles técnicas de persuasión colectiva" (Dader, 1990b, p. 223), periodo que precedió a una etapa -conocida como "de los efectos limitados", de 1940 a 1960 aproximadamente- en la que se consideró que los medios de comunicación solo tenían capacidad para reforzar o cristalizar opiniones y actitudes preexistentes en los individuos, quienes, por otra parte, hacían uso de una selectiva percepción (Lazarsfeld, 1985). Con posterioridad se inició un tercer momento, en el que resurgió la concepción de medios

\footnotetext{
4 El artículo se sustenta en el Proyecto de Investigación "Porteños Virtuales. Agenda Setting y otros efectos cognitivos", Programa de Reconocimiento Institucional de Investigaciones, Facultad de Ciencias Sociales (UBA), programación 2015-2017. El equipo de investigación está compuesto por Nayla Attas, Milagros Cabrejas, Soledad Camardo, Romina Damiani Ameri, Adrián Miranda, Sabrina Musolino, Javier Núñez, Florencia Perrotta, Facundo Rivero, Ana Laura Scaiano, Belén Sotelo, Belén Taquino y María Eugenia Tesio. Director: Daniel Cabrera.
} 
poderosamente influyentes, aunque con derivaciones pensadas desde una perspectiva socioestructural y cultural global, y no ya individual (Saperas, 1987). Se alude a cambios en la cognición y a transformaciones complejas y lentas, de mediano y largo plazo.

La aparición de la TV jugó un papel importantísimo en este sentido, por la masividad de su alcance y por la dependencia que generó. Dicha relevancia y eventual efecto pernicioso sobre el público fue duramente criticada (Sartori, 1992, 1998 y 2004), aunque también defendida con ahínco (Morduchowitz, 2004, 2005, 2008a y 2008b).

Paralelamente se desarrollaron otros tipos de estudios y de enfoques, entre los que sobresalen la teoría de la espiral del silencio (Noelle Neumann, 1995), según la cual el temor al aislamiento social conduce al individuo a ocultar aquellas opiniones que él percibe como minoritarias, no dominantes o en retroceso, y en la que los medios ocupan un importantísimo lugar en la apreciación de los sujetos respecto de la definición del clima de opinión predominante, y los estudios sobre recepción, que otorgan a las audiencias cierta competencia para decodificar los mensajes, independientemente de las intenciones de los mensajeros (Hall, 1973; Grimson y Varela, 1999; Rubin, 1996), y ponen el foco no tanto en el efecto de los medios sobre el público, sino en el uso que este hace de aquéllos.

A la vez, otros estudiosos realizaban especulaciones teóricas acerca de la formación de la opinión pública, basadas en la idea de opiniones inducidas por las elites que, mediante un encadenamiento en forma de cascada escalonada por una serie de remansos, decantaban en la masa luego de una serie de reformulaciones entre las que sobresalía el rol de los medios de comunicación masivos (Deutsch, citado por Sartori, 1990, p. 126).

En este contexto, el efecto agenda setting se materializa en una frase que luego se volvió emblema: "la prensa quizás no sea muy eficaz en decirle a la gente qué pensar, pero es asombrosamente 
exitosa en sugerirle en qué pensar" (Cohen, 1993, p. 13) y que dio lugar a la cuestión central de aquel cuerpo teórico ${ }^{5}$.

Esta conjetura enseguida pasó a constituir la primera y más importante fase de dicha teoría, ya que poco tiempo después los autores convinieron en que ciertos factores podían potenciar o inhibir el efecto de los medios, estableciéndose así la llamada fase dos (Aruguete, 2015, pp. 69-88; Casermeiro, 2003, pp. 101-120; McCombs, 2006, pp. 109-134).

Se trata de la intervención de variables -denominadas por los autores "condiciones contingentes"- que pueden potenciar o inhibir el efecto agenda y que se agrupan en seis dimensiones: 1) psicológicas: la necesidad de orientación de las audiencias, referida a la motivación presente en los individuos para conocer el mundo que los circunda, para lo que apelan a los medios; 2) conductuales: comunicación interpersonal y consumo de medios; 3) actitudinales: interés en la política y credibilidad en los medios; 4) demográficas: educación, ingresos, edad y sexo; 5) tipo de medios: gráficos o televisivos, y 6) tipo de temas: obstrusivos y no obstrusivos.

El desarrollo de la teoría siguió luego su curso, abordándose hasta el momento hasta tres fases más: la agenda de atributos o segundo nivel de la agenda setting (Casermeiro, 2003, p. 123; McCombs, 2006, p. 167); la construcción de la agenda, intermedia agenda o agenda building (Casermeiro, 2003, p. 151) y, finalmente, la emergencia de nuevos tipos de medios, digitales y redes sociales (Aruguete, 2015, p. 159). El cuerpo teórico sigue robusteciéndose con nuevas preguntas y derivaciones (McCombs, 2010).

El caso que se exhibe a continuación se refiere a la Ciudad Autónoma de Buenos Aires (CABA) y cuenta con mínimos antecedentes locales, todos ellos producidos en los últimos veinte años (Aruguete, 2015, pp. 53-58; Casermeiro, 2003, pp. 171-172).

Dader dice que el juego de palabras popularizado por Cohen (Cohen, 1993) había sido insinuado por Trenaman y McQuail dos años antes en TV and The Political Image (Dader, 1990a). 
La hipótesis que la investigación puso en juego postula que los medios poseen un significativo poder de trasladar sus preocupaciones al público, estableciendo como síntoma de dicha transferencia el establecimiento de agendas temáticas notablemente dependientes una de la otra, y proponiendo como metodología de contrastación la correlación estadística de las jerarquizaciones realizadas por los medios y por el público.

En consecuencia, el objetivo consistió en contrastar la denominada "fase uno" de la teoría de la agenda setting y, secundariamente, observar la incidencia de algunas de las condiciones contingentes indicadas en la fase dos.

\section{Soporte metodológico}

Si bien se trata de un procedimiento matemático avanzado, la correlación estadística que permite comparar las jerarquizaciones temáticas -correlación de rangos Rho de Spearman- resulta de aplicación e interpretación sencilla. Sin embargo, y a pesar de que la herramienta fue propuesta por los autores de la teoría desde el estudio inicial en Chapell Hill y aceptada por las más de 300 investigaciones que la replicaron, se advierte inmediatamente que el instrumento estadístico no implica causalidad, aunque provee importantísimas pistas para despejar la incógnita planteada.

Los resultados que se comentan más adelante se sostienen en una base empírica constituida por dos conjuntos de datos: una encuesta a 643 ciudadanos residentes en la Ciudad de Buenos Aires ("porteños"), basada en una muestra razonablemente representativa de dicha población, administrada del 8 al 12 de octubre de 2015, en el contexto del último tramo de la campaña electoral para elegir presidente de la nación ${ }^{6}$; y un relevamiento de nueve

Obsérvese que la investigación inicial de McCombs y Shaw (1972) alcanzó a cien (100) ciudadanos electoralmente indecisos, residentes en la localidad de Chapel Hill, Carolina del Norte (EEUU), que contaba con unos 26.200 habitantes en 1970 (Recurso electrónico disponible en: http:/ / www.ci.chapel-hill.nc.us). Por otra parte, 
diarios locales, pero de alcance nacional, realizado durante las seis semanas previas a la encuesta ${ }^{7}$, que permitió la colección de 20.050 registros temáticos extraídos de 23.005 noticias.

Es necesario resaltar que la muestra de medios se basó exclusivamente en diarios -versión papel- por dos motivos principales. El primero relacionado con la imposibilidad de construir una muestra de programas televisivos que pudiera ser relevada de hecho, para lo que deberíamos haber sorteado varios inconvenientes técnicos y de disponibilidad horaria del equipo de investigación -ni qué decir de medios radiofónicos o que se consumen a través de internet-.

El segundo, más importante, se basó en lo que señalan los propios investigadores de agenda setting, en cuanto a la mayor influencia de los diarios en detrimento de la televisión en la fijación de agenda (Canel, Llamas y Rey, 1996, p. 19; Casermeiro, 2003, pp. 81-88; Casermeiro, De la Torre y Téramo, 2009, p. 38; López Escobar, Llamas y Rey, 1996a, p. 19; López Escobar, Llamas y Rey, 1996b, p. 88) ${ }^{8}$, aunque en la investigación previa más consistente realizada en la Ciudad se obtuvo un resultado distinto (Caser-

ningún autor aclara explícitamente si sus muestras -a diferencia de la empleada por McCombs y Shaw en 1968, seleccionada aleatoriamente sobre un listado de votantes inscriptos- se basaran solo en electores indecisos; sin embargo, parece surgir, de las pocas precisiones al respecto, que la población estudiada estuvo siempre compuesta por la totalidad del cuerpo electoral (Casermeiro, 2003, pp. 78-89 y 182-183).

7 Cuatro a ocho semanas es el periodo óptimo indicado por la mayoría de los investigadores de agenda setting para otorgar posibilidad a los medios para surtir efecto (Casermeiro, 2003, pp. 211-218; y McCombs, 2006, pp. 92-100).

8 Por otra parte, la lectura de diarios no le va en zaga al consumo televisivo. Por un lado, circulan diariamente algo más de un millón de ejemplares de periódicos, mientras es posible suponer que cada ejemplar es leído por dos o más personas (Pautamas), sin considerar el uso a través de Internet. Asimismo, las encuestas desarrolladas por el Sistema Nacional de Consumos Culturales (Secretaría de Medios de Comunicación, 2006, p. 28) durante 2004-2006 establecieron que las proporciones de habitantes que se informan a través de la TV y de diarios es muy similar (alrededor del 52\% y 48\%, respectivamente), en tanto los datos de la encuesta de 2013 indican que el 83\% de los porteños escucha radio, el 74\% lee diarios y el 94\% mira TV (Sistema de Información Cultural de la Argentina, 2015). Por último, según la Encuesta Nacional de Hábitos de Lectura 2011, el 80\% de los argentinos lee el diario, aunque sea "de vez en cuando" (Consejo Nacional de Lectura, 2012, p. 7). 
meiro, 2003, p. 269). Asimismo, un tanto contradictoriamente, McCombs sostiene, por un lado, que al preguntarse "qué tipo de medio es más poderoso a la hora de establecer la agenda del público", la mejor respuesta es "depende", ya que varía mucho de una situación a otra (McCombs, 2006, p. 101); no obstante, casi inmediatamente indica que "en alrededor de la mitad de las ocasiones no hay una diferencia discernible entre los papeles como fijadores de agenda de los periódicos y de los telediarios. La otra mitad de las veces, los periódicos tienden a llevar ventaja en una proporción de aproximadamente 2 a 1" (McCombs, 2006, p. 102).

Además, algunos autores señalan incluso que la agenda de la TV está condicionada por la agenda de los diarios (López Escobar, Llamas y Rey, 1996b, p. 78).

De los 17 diarios que se imprimen en la Ciudad de Buenos Aires se elaboró una muestra de 9 de ellos, según se ve en el siguiente cuadro.

Periódicos editados en la Ciudad de Buenos Aires

\begin{tabular}{|l|l|}
\hline Elegidos para la muestra & Excluidos del relevamiento \\
\hline Ámbito Financiero & Argentinisches Tageblatt \\
\hline BAE Negocios & Buenos Aires Herald \\
\hline Clarín & El Argentino \\
\hline Crónica & Miradas al Sur \\
\hline Diario Popular & La Prensa \\
\hline El Cronista Comercial & La Razón \\
\hline La Nación & Olé \\
\hline Página 12 & Perfil \\
\hline Tiempo Argentino & \\
\hline
\end{tabular}

Fuente: elaboración propia.

Los periódicos incluidos en el relevamiento concentran algo más del 70\% de la cantidad semanal de ejemplares circulantes, 
calculada a partir de distintas fuentes en alrededor de 7.400.000 copias ${ }^{9}$.

Se excluyeron los diarios editados en idiomas distintos del español, destinados a un público compuesto mayoritariamente por extranjeros -Argentinisches Tablegatt y Buenos Aires Herald-. Los periódicos con temáticas específicas, únicas y distintas de lo político -el deportivo Olé-; los de tirada gratuita, que resultan una reproducción muy fiel de sus diarios madre -El Argentino y La Razón, básicamente apéndices de Tiempo Argentino y de Clarín, respectivamente-; y los que se editan solo los fines de semana y/o con una tirada muy escasa-Miradas al Sur, Perfil y La Prensa, respectivamente-.

Los aspectos asentados fueron: tema, número de páginas, tamaño, si se acompañaba con ilustración, fecha y sección.

Se muestra a continuación cómo se agruparon los temas más recurrentes.

Inseguridad: policía, servicio penitenciario, cárceles, agresiones, fuerzas de seguridad, homicidios, violencia, pedofilia, aborto, abuso, género y trata.

Economía: obras públicas, presupuesto, energía, exportaciones, AFIP, dólar, transporte, agro, inflación, Banco Nación, devaluación, empresas estatales, precios y servicios públicos.

Problemas sociales: accidentes, desarrollo social, inmigración, pobreza, protestas, asentamientos, desigualdad, tomas, urbanismo, vivienda, niñez, juventud y jubilados.

Educación: redes sociales, escuelas, universidad, tecnología, cultura, deportes y ciencia.

Salud: salud, drogadicción, obras sociales, hospitales.

\footnotetext{
Se consultaron siete fuentes (Ejes de Comunicación, Instituto Verificador de Circulaciones, Pautamas, Telam, TotalMedios, Sistema de Información Cultural de la Argentina y Wikipedia), encontrándose una disimilitud muy grande en cuanto a la cantidad de ejemplares informados como circulantes.
} 
Trabajo: desempleo, paritarias, gremios y salarios.

Política: campaña, partidos, elecciones, congreso, gobierno, medios, corrupción, justicia, juicios, coimas.

Seguidamente se procedió a ponderar la base en función de la tirada de cada diario, y según el día|momento de la semana -lunes a sábado, o domingo ${ }^{10}-$.

Finalmente, según sugerencias de McCombs (2006, p. 107) y Sierra Sánchez y Corbi (2013, pp. 5-7), se agregó a la ponderación indicada una puntuación extra a cada noticia, a partir de ciertas características de la publicación, que se detallan en el siguiente cuadro.

La encuesta permitió descubrir cuáles son los temas más importantes para la población al aplicar la denominada pregunta Gallup o MIP (Most Important Problem). Por otro lado, el análisis de contenido de las noticias seleccionadas posibilitó la identificación de un único tema por noticia. De esta forma se logró establecer la agenda del público y la agenda de los medios.

\section{Ficha técnica de la encuesta}

\begin{tabular}{|l|l|}
\hline Tipo de estudio & Encuesta coincidental \\
\hline Población & Electores de 16 años o más, residentes en la CABA \\
\hline $\begin{array}{l}\text { Instrumento de re- } \\
\text { colección de datos }\end{array}$ & $\begin{array}{l}\text { Cuestionario estructurado (60 variables, 40 preguntas), } \\
\text { con preguntas abiertas y cerradas con escalas ordina- } \\
\text { les, espontáneas, actitudinales, de clasificación y de } \\
\text { respuestas múltiples. Varias preguntas requerían la } \\
\text { visualización de tarjetas }\end{array}$ \\
\hline Diseño muestral & $\begin{array}{l}\text { Estratificado proporcional según comunas, con } \\
\text { selección de entrevistados ajustada según parámetros } \\
\text { poblacionales de sexo y edad (Censo 2010). Muestra } \\
\text { posponderada según voto anterior }\end{array}$ \\
\hline Cantidad de casos & 643 \\
\hline
\end{tabular}

10 La ponderación se realizó siguiendo los criterios señalados por Gervasoni y Fitz Herbert (2011). 


\begin{tabular}{|l|l|}
\hline $\begin{array}{l}\text { Margen de error } \\
\text { (bajo el supuesto } \\
\text { de una muestra } \\
\text { probabilística y una } \\
\text { confiabilidad del } \\
95 \%)\end{array}$ & 3.9 en más o en menos \\
\hline Fecha de campo & 8 a 12 de octubre de 2015 \\
\hline
\end{tabular}

Fuente: elaboración propia.

\section{Discutiendo agendas}

La encuesta a ciudadanos porteños permitió jerarquizar la lista de temas para ellos relevantes, que se observa a continuación junto con el porcentaje de menciones que recibió cada uno, y que constituye la agenda del público.

\section{Cuadro $\mathrm{N}^{\circ} 1$}

Agenda del público (en porcentajes)

\begin{tabular}{|l|l|l|}
\hline Temas & Encuestados & Respuestas \\
\hline INSEGURIDAD & 55.1 & 34.4 \\
\hline EDUCACIÓN & 27.0 & 16.9 \\
\hline ECONOMÍA & 26.6 & 16.6 \\
\hline SOCIALES & 17.2 & 10.7 \\
\hline POLÍTICOS & 13.2 & 8.2 \\
\hline TRABAJO & 8.3 & 5.2 \\
\hline SALUD & 6.7 & 4.2 \\
\hline OTROS & 4.8 & 3.0 \\
\hline NC & 1.1 & 0.7 \\
\hline TOTAL & $*$ & 100.0 \\
\hline
\end{tabular}

* El total no suma 100 debido a que los encuestados podían brindar hasta dos respuestas.

Base: total entrevistados (643). Fuente: elaboración propia.

Según se observa, básicamente cuatro temas acaparan la atención de los entrevistados: inseguridad, educación, economía y problemas sociales reúnen casi el $80 \%$ de las respuestas. 
Por otro lado, el ordenamiento de temas surgido del relevamiento de medios quedó constituido de la siguiente manera.

Cuadro $\mathrm{N}^{\circ} 2$

Agenda de los medios

\begin{tabular}{|l|c|}
\hline Temas & $\%$ \\
\hline Políticos & 39.0 \\
\hline Economía & 19.8 \\
\hline Inseguridad & 12.2 \\
\hline Educación & 6.1 \\
\hline Sociales & 5.6 \\
\hline Salud & 2.1 \\
\hline Trabajo & 1.5 \\
\hline Otros & 13.6 \\
\hline
\end{tabular}

Base: total noticias (23.005). Fuente: elaboración propia.

Seguidamente, se comparan ambas prioridades temáticas y se muestra el resultado de la aplicación del coeficiente de correlación de rangos Rho de Spearman.

\section{Cuadro $\mathrm{N}^{\circ} 3$}

Correlación entre prioridades temáticas*

\begin{tabular}{|l|l|l|}
\hline Temas & Medios & Público \\
\hline INSEGURIDAD & 3 & 1 \\
\hline EDUCACIÓN & 4 & 2 \\
\hline ECONOMÍA & 2 & 3 \\
\hline SOCIALES & 5 & 4 \\
\hline POLÍTICOS & 1 & 5 \\
\hline TRABAJO & 7 & 6 \\
\hline SALUD & 6 & 7 \\
\hline Rho de Spearman & 0.500 & \\
\hline
\end{tabular}

* En las prioridades de los medios no se consignan otros problemas (13,6\%), ni en las del público las correspondientes a los que no respondieron la pregunta o indicaron otros problemas (5.9\%).

Fuente: elaboración propia. 
Según se aprecia, se verifica una correlación positiva, pero que, aunque está muy cerca de serlo, no produce un resultado suficientemente robusto, según los parámetros que indica la bibliografía en la materia ${ }^{11}$. Además, la asociación tampoco resulta estadísticamente significativa.

Durante las seis semanas en que se desarrolló el relevamiento de diarios, los tres temas más mencionados se mantuvieron constantes: política, economía e inseguridad, en tanto los restantes mostraron distintos posicionamientos, pero a través de una firme tendencia, según se ve en el gráfico $\mathrm{N}^{\circ} 1$.

\section{Gráfico $\mathbf{N}^{\circ} 1$}

Variaciones en la jerarquización temática de los medios y evolución del coeficiente Rho de Spearman según semana de referencia.

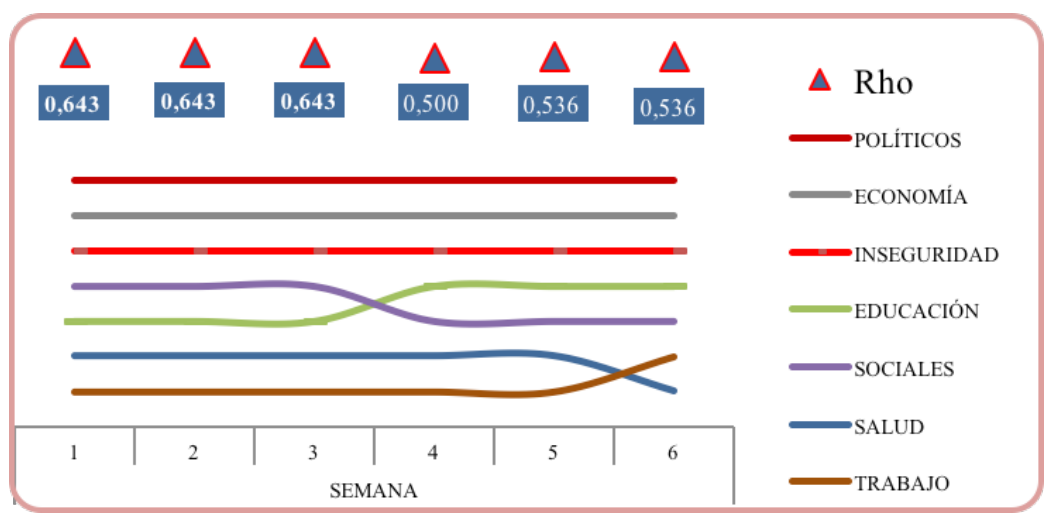

Fuente: elaboración propia.

En consecuencia, el coeficiente se mostró también muy estable, aunque los cambios en el orden de las jerarquizaciones implicaron una modificación en la significación del Rho.

\footnotetext{
11 “Según McCombs +.55 es el promedio estándar de correlación de los más de 300 estudios ya realizados (...) [mientras que] Rey Lennon señala que entre los estudios de agenda setting pueden considerarse como significativas las correlaciones a partir de $+.40^{\prime \prime}$ (Casermeiro, 2003, p. 77).
} 
En este sentido, lo hallado se opone en parte a lo consignado por Casermeiro (2003, p. 216), quien advierte en su estudio que los diarios, a diferencia de la TV, ejercen su efecto con más fuerza en la quincena más alejada de la medición de la agenda del público. Por su lado, McCombs (2006, pp. 92-100) indica que la influencia adquiere su mayor eficacia en un lapso de ocho semanas.

\section{Factores contingentes}

La investigación contrastó la incidencia de algunos factores contingentes. Para la Dimensión Actitudinal se hallaron valores significativos entre encuestados con gran interés en la política $(\mathrm{Rho}=.571)$ y alta confianza en los medios de comunicación $($ Rho=.714).

\section{Cuadro $\mathrm{N}^{\circ} 4$}

\section{Dimensión Actitudinal}

\begin{tabular}{|l|l|l|l|l|}
\hline Indicadores & \multicolumn{2}{|l|}{ Interés en la política } & \multicolumn{2}{l|}{ Credibilidad en los medios } \\
\hline Categorías & Bajo & Alto & Baja & Alta \\
\hline Rho Spearman & .500 & .571 & .393 & .714 \\
\hline
\end{tabular}

Fuente: elaboración propia.

Al respecto los antecedentes no son claros. Wanta, citado por Casermeiro (2003, p. 112), promueve la idea de una correlación alta entre interés en la política y el efecto agenda. Incluso sugiere que el interés político, al jugar un papel desencadenante de consumo mediático, estaría develando una relación espuria entre (alto) consumo y (alto) Rho.

En efecto, el análisis multivariado tipo Lazarsfeld -interés político como variable de control-indica que los consumidores de alta intensidad obtienen un Rho significativo únicamente cuando el interés político es bajo. Cuando este es alto, cualquiera sea la intensidad del consumo, la correlación no es robusta. 
Sin embargo, otros estudios encontraron resultados contrarios, que se explicaron a partir de dos argumentos. Los más interesados en política encuentran otros recursos informativos y no necesitan exponerse a los medios y, además, suelen "tener más desarrollados los mecanismos de defensa. Son más críticos a la jerarquización de la información, independientemente de la cantidad de información que consuman o del nivel de cobertura que reciban los temas" (Casermeiro, 2003, p. 113).

La confianza en los medios tampoco juega un papel claro en la bibliografía y parece imponerse el razonable argumento de que "aunque los individuos [sean] altamente críticos no pueden evitar contactarse con la cobertura que [los medios] hacen de ciertos temas" (Casermeiro, 2003, p. 115).

Dentro de la Dimensión Conductual se encontró un panorama algo más complejo ya que, por un lado, una baja consideración de la comunicación interpersonal produjo un coeficiente altamente positivo (Rho=.750) pero, por otro, la situación no resultó tan clara en cuanto a la intensidad del consumo.

En efecto, si bien un muy intenso consumo de medios produjo un coeficiente bastante mayor que un consumo de baja intensidad, ambos valores (Rho=.536 y Rho=.286, respectivamente) se sitúan por debajo de los estándares aceptados. Curiosamente, un comportamiento moderado en cuanto al uso de los medios obtiene un coeficiente significativo (Rho=.571).

Esta situación tal vez sea producto de la manera de categorizar la intensidad de consumo, variable no continua construida a partir de varios indicadores ${ }^{12}$.

12 El procedimiento de construcción se encuentra detallado en Cabrera, Camardo y Núñez (2016). 
Cuadro $\mathrm{N}^{\circ} 5$

Dimensión Conductual

\begin{tabular}{|l|l|l|l|l|l|}
\hline Indicadores & \multicolumn{4}{|c|}{ Comunicación interpersonal } & \multicolumn{3}{l|}{ Consumo de medios } \\
\hline Categorías & Baja & Alta & Bajo & Moderado & Alto \\
\hline Rho Spearman & .750 & .500 & .286 & .571 & .536 \\
\hline
\end{tabular}

Fuente: elaboración propia.

Por su parte, la Dimensión Demográfica aporta algo de coherencia a los datos, ya que consiguen valores más robustos los residentes en la zona norte de la ciudad, varones, de edades adultas (especialmente los de 51 a 65 años), con altos niveles de educación y que provienen de hogares con alto nivel socioeconómico (los ciudadanos de nivel bajo también obtienen un coeficiente significativo, aunque no tan elevado).

Además, las tendencias que pueden deducirse (mayor Rho a mayor edad y elevado nivel social, económico y educativo) coinciden con señalamientos de Casermeiro (2003, pp. 231 y 226-229), aunque se oponen a Canel, Llamas y Rey (1996, pp. 22-23).

\section{Cuadro $\mathrm{N}^{\circ} 6$}

\section{Dimensión Demográfica}

\begin{tabular}{|c|c|c|c|c|c|c|c|c|c|c|c|c|c|c|}
\hline \multirow{2}{*}{\begin{tabular}{|l|} 
Indicadores \\
Categorías
\end{tabular}} & \multicolumn{2}{|c|}{ Sexo } & \multicolumn{4}{|c|}{ Edad } & \multicolumn{3}{|c|}{$\begin{array}{l}\text { Zona de } \\
\text { residencia }\end{array}$} & \multicolumn{2}{|c|}{ Educación $^{13}$} & \multicolumn{3}{|c|}{$\begin{array}{c}\text { Nivel } \\
\text { socioeconómico }^{14}\end{array}$} \\
\hline & Varón & Mujer & $\begin{array}{l}\text { Hasta } \\
35\end{array}$ & $\begin{array}{l}36 a \\
50\end{array}$ & $\begin{array}{l}51 \mathrm{a} \\
65\end{array}$ & $\begin{array}{l}66 \text { y } \\
\text { más }\end{array}$ & Sur & Centro & Norte & Baja & Alta & Bajo & Medio & Alto \\
\hline $\begin{array}{l}\text { Rho } \\
\text { Spearman }\end{array}$ & .571 & .500 & .536 & .500 & .643 & .571 & .536 & .500 & .571 & .342 & .541 & .643 & .500 & .750 \\
\hline
\end{tabular}

Fuente: elaboración propia.

Finalmente, la Dimensión Tipo de Medio presenta características interesantes. Los diarios que mayor correspondencia mues-

\footnotetext{
13 Nivel Bajo: hasta secundario incompleto; Nivel Alto: secundario completo y más.

14 El nivel socioeconómico (de los hogares a los que pertenecen los encuestados) se construyó a partir de las indicaciones de la CEIM, SAIMO y AAM (2006).
} 
tran con la agenda del público son los denominados "populares", especialmente Diario Popular (Rho=.714). El diario Página 12 también obtiene un coeficiente alto (Rho=.679), lo mismo que, conjuntamente, los tradicionales Clarín y La Nación (Rho=.679), aunque no por separado. El resto de la prensa gráfica no consigue una asociación significativa, siendo los especializados en economía los que menos correlacionan con la agenda del público.

El caso curioso es el del diario Crónica, que presenta al tope de sus preferencias el tema inseguridad, del mismo modo que la agenda del público. No obstante, la correlación es negativa $(\mathrm{Rho}=.286)$ debido a la enorme falta de coincidencia en la jerarquización del resto de los temas.

\section{Cuadro $\mathrm{N}^{\circ} 7$}

\section{Dimensión Tipo de Medio (Diarios)}

\begin{tabular}{|l|l|l|l|l|l|l|l|l|l|}
\hline Categorías & Ámbito & BAE & $\begin{array}{l}\text { El } \\
\text { Cronista }\end{array}$ & $\begin{array}{l}\text { Popu- } \\
\text { lar }\end{array}$ & Crónica & $\begin{array}{l}\text { Página } \\
12\end{array}$ & Tiempo & Clarín & $\begin{array}{l}\text { La } \\
\text { Nación }\end{array}$ \\
\hline $\begin{array}{l}\text { Rho } \\
\text { Spearman }\end{array}$ & .214 & .214 & .286 & .714 & -.286 & .679 & .536 & .500 & .393 \\
\hline
\end{tabular}

Fuente: elaboración propia.

\section{Cuadro $\mathrm{N}^{\circ} 8$}

\section{Dimensión Tipo de Medio (Diarios agrupados)}

\begin{tabular}{|l|c|c|c|c|}
\hline Categorías & Económicos & Populares & Kirchneristas $^{15}$ & Tradicionales \\
\hline Rho Spearman & .214 & .750 & .500 & .679 \\
\hline
\end{tabular}

Fuente: elaboración propia.

Por otra parte, los lectores de diarios que alcanzan correlaciones más relevantes con la agenda de los medios son los que prefieren Clarín, La Nación y, sobre todo, los diarios especializados en economía.

\footnotetext{
15 Se agrupan bajo esta categoría diarios que durante la gestión kirchnerista mostraron un apoyo ostensible al gobierno nacional: Página 12 y Tiempo Argentino (O’Donnell, 2007; Ziblat, 2016).
} 
Cuadro $\mathrm{N}^{\circ} 9$

Correlación según diario que lee (Diarios)

\begin{tabular}{|l|l|l|l|l|l|l|}
\hline Categorías & No lee & Kirchneristas & Populares & Clarín & La Nación & Económicos \\
\hline Rho Spearman & .234 & .321 & .468 & .607 & .667 & .667 \\
\hline
\end{tabular}

Fuente: elaboración propia.

Dicho sea de paso, la correlación entre los lectores de un diario y la agenda de ese mismo diario opera como un indicador de la satisfacción del lector respecto de los temas elegidos por su diario preferido. Así, se observa que los que más deberían sentirse conformes serían los consumidores de los diarios económicos, seguidos por los de La Nación y Clarín.

Cuadro $\mathbf{N}^{\circ} 10$

Correlación entre el diario que lee y agenda del mismo diario (Diarios)

\begin{tabular}{|l|l|l|l|l|l|}
\hline Categorías & Kirchneristas & Populares & Clarín & La Nación & Económicos \\
\hline Rho Spearman & .321 & .523 & .607 & .631 & .667 \\
\hline
\end{tabular}

Fuente: elaboración propia.

\section{Nuevos factores contingentes}

Debido a que la investigación se desarrolló en un contexto político electoral -del mismo modo que una gran mayoría de estudios anteriores (Casermeiro, 2003) - resulta casi inevitable la consideración de "factores políticos" y partidarios en relación con el efecto de fijación de agenda. Por otra parte, los propios impulsores de la teoría destacan que "...las elecciones nacionales crean un laboratorio natural para el examen de los efectos mediáticos. Durante unas elecciones nacionales se da una descarga continua y masiva de mensajes referidos a los temas de interés público y a otros aspectos de la política. Y si esos mensajes producen algún tipo de efectos sociales significativos, estos se verificarán el día de las elecciones..." (McCombs, 2006, p. 116). 
En consecuencia, se midió la correlación de agendas a partir de cinco variables: intención de voto para presidente (primera vuelta), intención de voto en ballotage y opinión acerca de las gestiones de Cristina Fernández -presidenta en ejercicio- Mauricio Macri-jefe de gobierno saliente de la CABA y candidato opositor a presidente- y Daniel Scioli -gobernador saliente de la provincia de Buenos Aires y candidato oficialista a presidente-.

De esta manera, se observa que los votantes de Massa, Stolbizer y Macri, los que tienen opiniones negativas sobre las gestiones de los gobiernos nacional y provincial, y positiva sobre la gestión Macri, presentan correlaciones robustas, superiores a Rho=0.55. Dicho de otro modo, la agenda mediática se corresponde en mayor medida con las preferencias temáticas de los opositores al kirchnerismo.

Los resultados se sintetizan en los siguientes cuadros.

Cuadro $\mathrm{N}^{\mathrm{0}} 11$

Intención de voto a Presidente (primera vuelta)

\begin{tabular}{|l|c|c|c|c|c|c|c|}
\hline Categorías & Macri & Scioli & Massa & Stolbizer & Del Caño & En blanco & Abstención \\
\hline Rho Spearman & .607 & .536 & .714 & .643 & .072 & .667 & .286 \\
\hline
\end{tabular}

Fuente: elaboración propia.

\section{Cuadro $\mathrm{N}^{\mathrm{o}} 12$}

Intención de Voto a Presidente (segunda vuelta)

\begin{tabular}{|l|c|c|}
\hline Categorías & Macri & Scioli \\
\hline Rho Spearman & .714 & .500 \\
\hline
\end{tabular}

Fuente: elaboración propia.

Cuadro $\mathrm{N}^{\mathrm{o}} 13$

Opinión sobre las Gestiones de Gobierno

\begin{tabular}{|l|l|l|l|l|l|l|}
\hline Gestiones & \multicolumn{2}{l|}{ Cristina Fernández } & \multicolumn{2}{l|}{ Daniel Scioli } & \multicolumn{2}{l|}{ Mauricio Macri } \\
\hline Categorías & Positiva & Negativa & Positiva & Negativa & Positiva & Negativa \\
\hline Rho Spearman & .393 & .750 & .536 & .649 & .714 & .393 \\
\hline
\end{tabular}

Fuente: elaboración propia. 
Por último, el estudio examinó el efecto agenda a la luz de un "factor mediático", que contempló el tipo de consumo de medios que realizó el residente porteño. En función de las particularidades de consumo que exhibían los ciudadanos en un estudio previo, caracterizadas por su intensidad, diversidad, extensión y, a la vez, desconfianza hacia los medios (Cabrera, 2015), en esta oportunidad se construyó una herramienta metodológica que permitió estructurar el tipo de consumo mediático a partir de la intensidad y de los medios preferentemente empleados por los porteños para nutrirse de información política.

El instrumento, cuya elaboración se detalla y explica en Cabrera, Camardo y Núñez (2016), posibilitó tipificar la rutina de información de los porteños, emergiendo dos tipos básicos de consumidores: tradicionales y pantalleros. Los primeros, basados en la lectura de diarios en papel y, en menor medida, en la escucha de radio; los segundos, sustentados en la utilización del soporte pantalla: sobre todo, televisión e internet.

En cuanto a la intensidad, se segmentó en tres posibilidades: alta, moderada y baja.

Por último, la combinación de ambas dimensiones posibilitó la construcción de una variable con nuevas categorías, distinguiendo el medio preferido -diario, televisión o internet- y la intensidad de consumo en cada uno -alto, moderado o bajo ${ }^{16}$.

Una vez calculados los coeficientes, se observó correlaciones significativas para los consumidores tradicionales y de intensidad moderada, y, más específicamente, para los diarieros de intensidad alta, televidentes moderados y todos los internautas.

Los siguientes cuadros resumen lo dicho.

16 Se excluyó la radio de esta clasificación por no contar con indicadores suficientes para categorizar la intensidad de la escucha. 
Cuadro ${ }^{\circ} 14$

Tipo de consumo (resumido)

\begin{tabular}{|l|l|l|}
\hline Categorías & Tradicionales & Pantalleros \\
\hline Rho Spearman & .679 & .541 \\
\hline
\end{tabular}

Fuente: elaboración propia.

\section{Cuadro $\mathrm{N}^{\circ} 15$}

Tipo de consumo (detallado)

\begin{tabular}{|l|l|l|l|l|}
\hline Categorías & Diarieros & Radioescuchas & Televidentes & Internautas \\
\hline Rho Spearman & .750 & .571 & .270 & .500 \\
\hline
\end{tabular}

Fuente: elaboración propia.

\section{Cuadro $\mathrm{N}^{\mathrm{o}} 16$}

\section{Intensidad del consumo}

\begin{tabular}{|l|l|l|l|}
\hline Categorías & Bajo & Moderado & Alto \\
\hline Rho Spearman & .286 & .571 & .536 \\
\hline
\end{tabular}

Fuente: elaboración propia.

\section{Cuadro $\mathrm{N}^{\circ} 17$}

Tipo e intensidad de consumo

\begin{tabular}{|l|l|l|l|l|l|l|l|l|l|}
\hline \multirow{2}{*}{ Categorías } & \multicolumn{3}{|l|}{ Diariero } & \multicolumn{3}{l|}{ Televidente } & \multicolumn{3}{l|}{ Internauta } \\
\cline { 2 - 10 } & Bajo & Moderado & Alto & Bajo & Moderado & Alto & Bajo & Moderado & Alto \\
\hline Rho Spearman & .500 & .468 & .750 & .536 & .571 & .107 & .607 & .571 & .595 \\
\hline
\end{tabular}

Fuente: elaboración propia.

\section{Conclusiones}

Estrictamente, la hipótesis del efecto agenda no pudo ser validada en este estudio, teniendo en cuenta una interpretación rígida de las recomendaciones de los investigadores más reconocidos.

Sin embargo, las circunstancias que rodean ese dato tan relevante configuran un panorama muy consistente y que pueden 
explicar, por lo menos parcialmente, el significado del consumo de medios que tienen los porteños, incluyendo cómo aquellos impactan sobre estos.

El contexto de los datos implica situarse en un ambiente altamente politizado, signado por la campaña electoral presidencial, y también por un $54 \%$ de porteños que se manifestaron simpatizantes o adherentes a partidos o dirigentes políticos, según se advierte en otro de los estudios emanados de la investigación que soporta este artículo (Cabrejas, Cabrera, Damiani Ameri y Scaiano, 2016). Además, el consumo de medios de los ciudadanos porteños se reveló extremadamente intenso y a la vez muy desconfiado. Según se lee en Cabrera, Camardo y Núñez (2016), el $98 \%$ de los porteños se informa sobre política y dos tercios lo hacen a través de más de un medio; por otra parte, solo $21 \%$ cree que los medios son confiables pero el doble piensa que no lo son.

Las dimensiones actitudinales, conductuales y demográficas de los factores contingentes permiten advertir que quienes obtienen una correlación significativa, que daría sustento a la hipótesis de la agenda setting, conforman un sector social acotado y bien definido: se trata de ciudadanos con alto interés en la política, gran credibilidad en los medios de comunicación -aunque moderado consumo de medios y baja valoración de la comunicación interpersonal como canal para alcanzar la información políticay, a la vez, varones adultos, de alto nivel educativo y socioeconómico, residentes en las zonas más cotizadas de la ciudad y consumidores del diario La Nación y de los especializados en materia económica.

La perspectiva se completa atendiendo a nuevos factores contingentes -políticos y mediáticos-, que indican que los ciudadanos opositores al kirchnerismo, usuarios de internet e intensos consumidores de diarios son los que registran mayores coincidencias entre sus agendas y las de los medios de comunicación.

Ambos datos llaman la atención. El perfil social, económico, demográfico y político de los segmentos que presentan una jerar- 
quización temática con mayor correspondencia respecto de los medios, responde a la configuración ideal para constituirse en el eslabón de oro entre medios y fracciones ciudadanas menos interesadas en política y con características opuestas en las restantes dimensiones.

Podría evocarse, sin forzar demasiado la conexión, la teoría de la comunicación en dos etapas (Lazarsfeld y otros, 1962, p. 211) y el rol que este autor les asignó a los líderes de opinión en su famoso estudio (Lazarsfeld y otros, 1962, p. 91). Aunque en la era de las comunicaciones, y sobre todo en la etapa digital, el contacto entre líderes y no líderes no opera como hace 70 años, los líderes se parecen a las brujas: nadie cree en ellos, "pero que los hay, los hay". Así, la información de los medios llegaría a los menos interesados en política a través de la versión de los más interesados.

La hipótesis es congruente con un enfoque asentado en el descubrimiento de cierta brecha entre los intereses periodísticos y las preocupaciones ciudadanas: lo que los medios publican en lugares destacados se distancia de lo que el público busca informativamente (Boczkowski y Mitchelstein, 2015).

No obstante, los investigadores en agenda setting, aun asignando a aquel grupo social -especialmente a los más educados- mayor susceptibilidad "a que los medios delimiten la importancia de los temas" (Aruguete, 2015, p. 71), invocaron -de la mano de la teoría de Knowledge Gap (Baquerín, 2008)- una mejor preparación de estos individuos "para entender el significado de los contenidos de los medios" (Casermeiro, 2003, p. 111), principal argumento esgrimido para eximirlos de poseer un pensamiento a salvo de la influencia mediática. En verdad, esta independencia de criterio alcanza también a todos los segmentos ya que, como quedó demostrado, los no líderes tampoco basan su agenda en la de los medios. Y habría que decir, realmente, mucho menos que lo líderes.

De todas formas, los líderes parecen jugar un rol especial. No solo ocupan un lugar preferente en el ya mencionado mo- 
delo de formación de opiniones en cascada (Sartori, 1990), sino que, debido a que los medios de comunicación tienen una relación muy estrecha con elites económicas, sociales y políticas, “el mundo es -para el público en general- el mensaje de los medios de comunicación. Si los medios no son tan persuasivos como se teme, ello se debe en no poco a que el próximo, y verdadero gran salto de la cascada, incide en, y es mediado por, los creadores de opinión locales. Estos forman el estrato políticamente atento de una población, del 5 al 10 por 100 del público..." (Sartori, 1990, pp. 127-128), y "pueden bloquear o reforzar, disminuir o ampliar y seleccionar, en cualquier caso, la importancia de, y otorgar credibilidad a, los mensajes de los medios..." (Sartori, 1990, p. 128).

Si fueran simples borboteos, explica Sartori aludiendo a la cascada, el propio modelo los explicaría; sin embargo -continúa-, el brote de opiniones a veces toma la forma de un oleaje irresistible que se convierte en "corrientes de opinión" (Sartori, 1990, p. 128), a punto tal que el autor, a partir de esta habilidad de los líderes, basada en una concurrencia de factores -dentro de la que sobresale la "expansión masiva de la educación superior" (Sartori, 1990, p. 129)-, les confiere gran protagonismo en lo que él denomina un segundo modelo de formación de opiniones.

En síntesis, podría conjeturarse que el ciudadano poco interesado en política presta escasa atención a lo que, sobre política, dicen los medios; en cambio, a los líderes les importa mucho lo que los medios publican porque, entre otros motivos, los sienten competidores de su función de persuasores ciudadanos, en la que vienen perdiendo crédito notablemente.

En consecuencia, la transferencia de relevancia que propone la agenda setting se ve mediada, al menos en algunos casos, por actores sociales y políticos con una configuración bien determinada, escaso peso demográfico y alto compromiso y gravitación política: los líderes de opinión. 


\section{Referencias}

Aruguete, N. (2015). El poder de la agenda: política, medios y público. Buenos Aires: Biblos.

Baquerín, M. T. (2008). Los desniveles del conocimiento. En T. Baquerín (Ed.), Los medios, ¿aliados o enemigos del público? Buenos Aires: EDUCA.

Boczkowski, p. y Mitchelstein, E. (2015). La brecha de las noticias. La divergencia entre las preferencias informativas de los medios y el público (The News Gap. When the Information Preferences of the Media and the Public Diverge, 2013). Buenos Aires: Manantial.

Cabrejas, M., Cabrera, D., Damiani Ameri, R. y Scaiano, A. L. (2016). El heterogéneo votante porteño. Identidades políticas y consumo de medios. XII Congreso Nacional y $V$ Internacional sobre Democracia. Universidad Nacional de Rosario, Rosario.

Cabrera, D., Camardo, S. y Núñez, J. (2016). Tipología del consumidor mediático. Características socioeconómicas, preferencias políticas y prioridades temáticas. XII Congreso Nacional y $V$ Internacional sobre Democracia. Universidad Nacional de Rosario, Rosario.

Cabrera, D. (2015). Informados y Desconfiados. Los porteños se defienden de la agenda setting. XII Congreso Nacional de Ciencia Política. Sociedad Argentina de Análisis Político (SAAP), Mendoza.

Cabrera, D. (2011). El rol de los medios, las formas de la opinión pública y las estrategias comunicacionales en la campaña electoral de 2011. Lo que vendrá, 8(6), Carrera de Ciencia Política (UBA), Buenos Aires.

Cabrera, D., Camardo, S. y Núñez, J. (2016). Tipología del consumidor mediático. Características socioeconómicas, preferencias políticas y prioridades temáticas. $V$ Encuentro Latinoamericano de Metodología de las Ciencias Sociales (ELMECS). Red Latinoamericana de Metodología de las Ciencias Sociales (RedMet), Mendoza.

Canel, M. J., Llamas, J. p. y Rey, F. (1996). El primer nivel del efecto agenda-setting en la información local: los 'problemas más importantes' de la ciudad de Pamplona. Comunicación y Sociedad, IX(1- 2), Universidad de Navarra. 
Casermeiro, A. (2003). Los medios en las elecciones. Agenda Setting en la Ciudad de Buenos Aires. Buenos Aires: Editorial de la Universidad Católica (EDUCA).

Casermeiro, A., De la Torre, L. y Téramo, M. T. (2009). Buenos Aires elige presidente: un estudio en el marco de la teoría de la agenda setting. Buenos Aires: EDUCA.

Cohen, B. (1993). The Press and Foreing Policy (1963). Princeton University Press.

Dader, J. L. (1990a). La canalización o fijación de la agenda por los medios. En A. Muñoz Alonso y otros. Opinión pública y Comunicación politica. Madrid: EUDEMA.

Dader, J. L. (1990b). La evolución de las investigaciones sobre la influencia de los medios y su primera etapa: teorías del impacto directo. En A. Muñoz Alonso y otros. Opinión Pública y comunicación política. Madrid: EUDEMA.

Gervasoni, C. y Fitz Herbert, A. (2011). Monitoreo de Publicidad Electoral y Publicidad Oficial durante la Campaña Electoral 2011. Informe II: Campaña para las Elecciones Nacionales (14 a 22 de octubre de 2011). Buenos Aires: Departamento de Ciencia Política y Estudios Internacionales, Universidad Torcuato Di Tella (UTDT).

Grimson, A. y Varela, M. (1999). Audiencias, cultura y poder. Estudio sobre televisión. Buenos Aires: EUDEBA.

Hall, S. (1973). Encoding and Decoding in the Television Discourse. Birmingham: Centre for Contemporary Cultural Studies.

Kitzberger, Ph. (2012). "La madre de todas las batallas": el kirchnerismo y los medios de comunicación. En A. Malamud y M. De Luca (Comp.), La politica en tiempo de los Kirchner. Buenos Aires: EUDEBA.

Landi, O., Vacchieri, A. y Quevedo, L. (1990). Públicos y consumos culturales de Buenos Aires. Buenos Aires: Centro de Estudios de Estado y Sociedad (CEDES).

Lazarsfeld, p. (1985). La campaña electoral ha terminado (The Election is Over, 1953). En M. De Moragas (Ed.), Sociología de la comunicación de masas. Barcelona: Gustavo Gilli.

Lazarsfeld, P., Berelson, B. y Gaudet, H. (1962). El pueblo elige. Estudio del proceso de formación del voto durante una campaña electoral (The Peoples's Choice. How the Voter Makes up his Mind in a Presidential Campaign, 1944). Buenos Aires: Ediciones 3. 
López Escobar, E., Llamas, J. p. y Rey, F. (1996a). El primer nivel del efecto agenda-setting en la información local: los "problemas más importantes" de la ciudad de Pamplona. Comunicación y Sociedad, IX(1-2), Universidad de Navarra.

López Escobar, E., Llamas, J. p. y Rey, F. (1996b): La agenda entre los medios: primero y segundo nivel. Comunicación y Sociedad, IX(1-2), Universidad de Navarra.

McCombs, M. (2006). Estableciendo la agenda (Setting the Agenda, 2004). Barcelona: Paidós.

McCombs, M. (2010). Extending our theoretical maps: psychology of agenda-setting. Central European Journal of Communication, 2, University of Wroclaw.

McCombs, M. \& Shaw, D. (1972). The Agenda Setting function of Mass Media. Public Opinion Quarterly, 36, Oxford University Press.

Morduchowitz, R. (2004). Medios y violencia social. Clarín, Buenos Aires, 5 de octubre.

Morduchowitz, R. (2005). Un debate para todos. La Nación, Buenos Aires, 8 de julio.

Morduchowitz, R. (2008a). La generación multimedia. Significados, consumos y prácticas culturales de los jóvenes. Buenos Aires: Paidós.

Morduchowitz, R. (Coord.). (2008b). Los jóvenes y las pantallas. Buenos Aires: Gedisa.

Neumann N. (1995). La espiral del silencio. Barcelona: Paidós.

O’Donnell, M. (2007). Propaganda K. Buenos Aires: Planeta.

Sartori, G. (1990). La democracia gobernada y la democracia gobernante. En G. Sartori, Teoría de la democracia, Tomo I. Buenos Aires: Rei Argentina.

Sartori, G. (1992). Videopoder. En G. Sartori, Elementos de Teoría Política. Madrid: Alianza.

Sartori, G. (1998). Homovidens. La sociedad teledirigida. Madrid: Taurus.

Sartori, G. (2004). Tiene futuro la democracia. Ñ, Buenos Aires, 13 de noviembre.

Schuliaquer, I. (2014). El poder de los medios. Buenos Aires: Capital Intelectual.

Sierra Sánchez, J. y Corbi, C. (2013). Análisis del tratamiento informativo sobre la "toma de Bangkok (2010)" en los diarios El País y El Mundo. Ámbitos, 22, Universidad de Sevilla. 
Rubin, A. (1996). Usos y efectos de los media: una perspectiva uso-gratificación. En J. Bryant y D. Zillmann (Comp.), Los efectos de los medios de comunicación. Investigaciones y teoría (Media Effects. Advances in Theory and Research, 1994). Buenos Aires: Paidós.

Saperas, E. (1987). Los efectos cognitivos de la comunicación de masas. Barcelona: Ariel.

Ziblat, G. (2016). “No existe el periodismo independiente", dice el dueño del diario Página 12", Perfil, Buenos Aires, 28 de febrero. Recuperado de http://www.perfil.com/mobile/?nota=/ contenidos/2016/02/27/noticia_0145.html

Zunino, E. (2014). La cobertura mediática del 'conflicto campo-gobierno' de 2008 en la prensa gráfica argentina (Tesis de Maestría). Universidad Nacional de Quilmes, Buenos Aires.

\section{Otras fuentes de datos}

Cámara de Empresas de Investigación Social y de Mercado (CEIM), Sociedad Argentina de Investigadores de Marketing y Opinión (SAIMO) y Asociación Argentina de Marketing (AAM). (2006). NSE 2006. Buenos Aires.

Consejo Nacional de Lectura (2011). Encuesta Nacional de Hábitos de Lectura. Recuperado de http: / / sinca.cultura.gov.ar/archivos / documentos/ENHL_2011.pdf

Instituto Verificador de Circulaciones (IVC). Recuperado de http:/ / www.ivc.org.ar Pautamas. Recuperado de http://www. pautamas.com.

Secretaría de Medios de Comunicación, Jefatura de Gabinete de Ministros, Presidencia de la Nación (2006). Sistema Nacional de Consumos Culturales, noviembre 2006, Buenos Aires. Recuperado de http://industriasdecontenido.files.wordpress. com/2010/08/ic-arg-sistema-nacional-de-consumos-culturales-3-20061.pdf

Sistema de Información Cultural de la Argentina (2015). Encuesta Nacional de Consumos Culturales 2015. Buenos Aires: Dirección Nacional de Industrias Culturales, Ministerio de Cultura, Presidencia de la Nación. Recuperado de http: / / sinca.cultura.gob.ar/sic/publicaciones/libros/EECC.pdf

TotalMedios. Recuperado de http:/ / www.totalmedios.com 